\title{
Utilisation d'un taux d'incidence mobile sur 40 jours pour surveiller la coqueluche en Nouvelle-Écosse en 2015
}

\author{
J Born ${ }^{1,2 *}$, A Coombs $^{1,4}$, V Ryan², M LaFreniere ${ }^{2,3}$, L Earle $^{2}$, S Fleming ${ }^{2}$, A Fitzgerald ${ }^{2}$, F Atherton ${ }^{2}$
}

\section{Résumé}

Contexte : La coqueluche peut causer une grave infection bactérienne des voies respiratoires, particulièrement chez les nourrissons. Entre le 1er janvier et le 31 décembre 2015, il y a eu une augmentation du nombre de cas de coqueluche déclarés en Nouvelle-Écosse. Les pratiques de surveillance liées à la coqueluche en Nouvelle-Écosse ont été exigeantes puisque les données sur la couverture vaccinale n'étaient pas disponibles et les renseignements sur les taux n'étaient ni opportuns ni précis en ce qui touche la géographie. Les responsables de la santé publique de la Nouvelle-Écosse ont décidé d'adopter une nouvelle technique de surveillance pour éclairer les mesures de santé publique dans l'ensemble de la province.

Objectif : Évaluer l'utilisation d'un taux d'incidence mobile sur 40 jours afin de surveiller l'activité de la coqueluche en Nouvelle-Écosse.

Intervention : Un taux d'incidence mobile sur 40 jours a été calculé pour la coqueluche selon les groupes d'âge et les différentes échelles géographiques. Les autorités sanitaires publiques ont continué d'anticiper de nouveaux cas de coqueluche si les contacts de cas connus se faisaient toujours pendant la période d'incubation (intervalle de six à 20 jours). On a choisi la période d'incubation de 40 jours afin de refléter la limite supérieure de la période d'incubation. Les taux ont été calculés à partir des projections de population de Statistique Canada pour 2014, puis comparés aux nombres de cas traditionnels et aux incidences cumulatives. Des décideurs de la santé publique ont évalué l'utilité des statistiques.

Résultats: On a noté une activité accrue de la coqueluche dans l'ensemble de la Nouvelle-Écosse, notamment dans la région Sud-Ouest. L'utilisation d'un taux d'incidence mobile sur 40 jours comme outil de surveillance a engendré des descriptions plus opportunes et précises sur le plan géographique des tendances actuelles de l'activité de la coqueluche et a contribué à la détermination de mesures de santé publique appropriées. Les responsables de la santé ont apprécié les renseignements provenant du taux d'incidence mobile puisqu'ils leur ont permis de gérer les activités en fonction d'estimations hebdomadaires à différentes échelles géographiques.

Conclusion : Le taux d'incidence mobile s'est avéré un outil utile pour surveiller une augmentation clinique des cas de coqueluche en Nouvelle-Écosse et orienter les mesures de santé publique connexes. Le taux d'incidence mobile fourni a généré des renseignements précis sur le plan géographique et opportuns qui ont été utiles pour estimer les nouveaux cas en l'absence d'information fiable sur la couverture vaccinale. Cette méthode pourrait compléter la surveillance épidémiologique traditionnelle des maladies transmissibles, en particulier celles qui se caractérisent par de longues périodes d'incubation et de faibles nombres de cas.

\section{Affiliations}

${ }^{1}$ Centre pour l'infrastructure en santé publique, Agence de la santé publique du Canada, Ottawa (Ontario)

${ }^{2}$ Ministère de la Santé et du Mieux-être de la Nouvelle-Écosse, Halifax (Nouvelle-Écosse)

${ }^{3}$ Centre de la lutte contre les maladies transmissibles et les infections, Agence de la santé publique du Canada, Ottawa (Ontario)

${ }^{4}$ Autorité sanitaire de la Nouvelle-Écosse, Kentville (Nouvelle-Écosse)

*Correspondance : jennifer. born@forces.gc.ca

Citation proposée : Born J, Coombs A, Ryan V, LaFreniere M, Earle L, Fleming S, Fitzgerald A, Atherton F. Utilisation d'un taux d'incidence mobile sur 40 jours pour surveiller la coqueluche en Nouvelle-Écosse en 2015. Relevé des maladies transmissibles au Canada. 2017;43(2):54-60. https://doi.org/10.14745/ccdr.v43i02a03f

\section{Introduction}

La coqueluche (toux coquelucheuse) est une infection respiratoire très contagieuse causée par la bactérie Bordetella pertussis. Elle est endémique dans la population humaine et difficile à maîtriser, partiellement en raison d'une longue période de transmissibilité (de six à 20 jours) (1-3), de la sous-immunisation de la population et du déclin évident de l'immunité conférée par le vaccin. La maladie peut être particulièrement grave chez les enfants de moins de 12 mois (1), 
les complications comprenant la pneumonie, les convulsions, I'encéphalopathie, les hernies et la mort (4). Des formes bénignes de la maladie peuvent se manifester chez les adultes et les adolescents, qui sont moins susceptibles de recevoir un diagnostic et de se faire traiter $(5,6)$. Les données indiquent que les enfants accusent un retard dans la vaccination $(7,8)$ et que l'immunité après la vaccination peut décliner au fil du temps $(9,10)$, ce qui donne lieu à une population vulnérable qui contribue à un réservoir continu de coqueluche chez les humains $(11,12)$.

L'activité de la coqueluche peut être de nature cyclique (13-15).

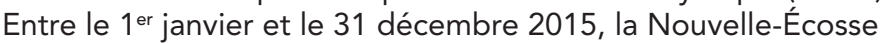
a connu une augmentation du nombre de cas de coqueluche. Cette situation a créé la nécessité de surveiller de plus près et d'analyser l'activité de la coqueluche dans la province. La structure du système de santé de la Nouvelle-Écosse comprend deux autorités sanitaires : I'autorité sanitaire de la Nouvelle-Écosse (ASNE) (subdivisée en quatre zones de gestion locales) et l'autorité sanitaire Izaak Walton Killam (IWK). Conformément aux recommandations du Canada et de l'Organisation mondiale de la Santé en matière de surveillance de la coqueluche $(16,17)$, les données sur les maladies à déclaration obligatoire sont déclarées mensuellement à l'échelle des zones et des provinces et sur une base annuelle à l'échelle provinciale.

Au cours de l'éclosion de coqueluche de 2015, les rapports de surveillance mensuels et annuels n'ont pas pleinement répondu aux besoins en information des autorités locales en matière de santé publique afin de réagir au nombre de cas de coqueluche à la hausse. Comme la Nouvelle-Écosse est une petite province (population totale de moins d'un million), les rapports fréquents sur l'activité de la coqueluche ( $p$. ex. hebdomadaires) et par zone de santé (ou géographies inférieures) posent des difficultés en raison des faibles nombres de cas. Les résultats sont statistiquement moins fiables lorsque les activités de la maladie sont rares (18), et la déclaration de faibles nombres peut menacer la confidentialité, en particulier dans les populations plus petites $(19,20)$.

D'autres options ont été envisagées afin d'atténuer les défis que présentent les petits nombres. L'incidence cumulative à ce jour a été jugée problématique puisqu'elle ne reflétait pas avec exactitude l'activité actuelle de la maladie. Comme I'incidence cumulative a été calculée à partir d'un dénominateur statique, la ligne de tendance a simplement augmenté toutes les semaines, de sorte qu'elle n'a pas révélé d'augmentations ou de diminutions d'une semaine à l'autre. Le taux d'attaque a également été pris en compte, mais les données insuffisantes sur la couverture vaccinale ont compliqué la quantification des populations réceptives.

Les autorités en matière de santé publique ont décidé d'essayer un taux d'incidence mobile sur 40 jours. Le choix s'est arrêté sur l'intervalle de 40 jours afin de refléter de façon prudente deux fois la limite supérieure de la période d'incubation de six à 20 jours. On a recouru aux estimations mobiles dans les situations d'éclosion (21), souvent sous la forme d'une moyenne mobile de cinq ans arbitraire afin de définir l'activité normale ou d'établir des bases de référence à des fins comparatives. Pour autant que les auteurs le sachent, il s'agit de la première utilisation documentée d'un taux d'incidence mobile élaboré afin de rendre compte des caractéristiques propres à la maladie (c.-à-d. la période d'incubation) et de prévoir l'activité de la maladie.

L'objectif de cet article est de décrire la première utilisation d'un taux d'incidence mobile sur 40 jours afin de surveiller l'activité de la coqueluche et d'évaluer si cette méthode a aidé à orienter les mesures de santé publique. Cette méthode a complété la surveillance de routine durant une augmentation de l'activité de la coqueluche en Nouvelle-Écosse entre le $1^{\text {er }}$ janvier et le 31 décembre 2015.

\section{Intervention}

\section{Sources des données}

\section{Cas confirmés de coqueluche déclarés selon leur date d'épisode}

L'application pour la surveillance des maladies à déclaration obligatoire (ASMDO) a été utilisée pour la surveillance continue des cas de coqueluche en Nouvelle-Écosse qui correspondaient aux définitions de cas à l'échelle provinciale (confirmés, probables et soupçonnés) (22). Des renseignements géographiques (dont l'âge et la région géographique de résidence) ont également été extraits de I'ASMDO à l'aide de COGNOS, une trousse de logiciels commerciaux d'extraction, d'analyse et de production de rapports. Cette information a servi à calculer le nombre de cas de coqueluche confirmés selon leur date d'épisode déclarée par mois et région géographique. La date d'épisode faisait le plus souvent référence à la date d'apparition des symptômes. La date du diagnostic clinique a été utilisée dans les cas où la date d'apparition n'était pas disponible et la date de prélèvement des échantillons, dans les cas où ni la date d'apparition ni la date du diagnostic clinique n'étaient disponibles. La date d'obtention du résultat des tests en laboratoire a été utilisée seulement lorsqu'aucune autre date n'était disponible (23).

\section{Analyse des données}

\section{Statistiques traditionnelles}

Les cas déclarés entre le 1er janvier 2015 et le 31 décembre 2015 ont été extraits mensuellement de l'ASMDO à l'aide de COGNOS. Les statistiques traditionnelles, dont les nombres mensuels et le taux par tranche de 100 000, ont été calculées mensuellement pour la province dans son ensemble et par zone.

\section{Incidence mobile}

Les cas rétrospectifs de 2015 et des 40 derniers jours de 2014 ont servi à calculer les taux d'incidence mobile sur 40 jours pour toute l'année 2015 (selon la date d'épisode). Les cas confirmés déclarés entre le 21 novembre 2014 et le 31 décembre 2015 ont été extraits de l'ASMDO à l'aide de COGNOS et ont servi à calculer les taux d'incidence mobile sur 40 jours pour 2015.

Pour chaque jour civil de 2015, l'incidence de la coqueluche a été calculée pour les 40 jours précédents selon la date d'épisode déclarée. Le choix s'est arrêté sur le taux d'incidence sur 40 jours 
parce qu'il représentait deux fois la limite extérieure du nombre de jours pour l'incubation typique de la coqueluche (intervalle de six à 20 jours) (1,3). Sur la base d'une période d'incubation maximale moyenne de 20 jours, on a décrété qu'un cas et tous les contacts pertinents étaient non infectieux 40 jours après la date d'épisode du cas.

On a défini l'incidence mobile (MI) comme la somme des cas (A) au cours des $\mathrm{n}$ jours précédents divisée par la personne-temps (PT) (équation mathématique disponible sur demande).

On a supposé que les populations étaient statiques et toutes à risque. Ainsi, chaque personne dans les populations respectives a compté pour une unité de temps dans le dénominateur. L'incidence mobile a été calculée pour chaque jour civil ( $t$ ) en additionnant le nombre de cas de coqueluche (A) au cours des 40 jours précédents sur la personne-temps. Les taux ont été calculés dans Stata (24) avec les données démographiques de 2014 de Statistique Canada (25). Les taux pour 100000 habitants ont été calculés à différents niveaux géographiques, y compris quatre zones de gestion de la santé provinciales et la région géographique du Sud-Ouest - une sous-section de la zone Ouest qui inquiétait les autorités sanitaires locales.

\section{Évaluation de l'utilité des méthodes traditionnelles et du taux d'incidence mobile sur $\mathbf{4 0}$ jours}

Les méthodes de l'incidence mobile découlent d'un processus itératif. Pour compléter les rapports mensuels et annuels, l'équipe d'évaluation et surveillance de la santé de la population a d'abord créé un rapport hebdomadaire qui comprenait les nombres de cas de coqueluche par zone et groupe d'âge. Ces données ont été diffusées au personnel de santé publique, aux médecins hygiénistes, aux épidémiologiques et aux gestionnaires des maladies transmissibles au ministère de la Santé et du Mieux-être provincial et aux niveaux locaux (ASNE) du système de santé publique. Le taux d'incidence mobile a été mis au point comme statistique additionnelle d'après la rétroaction reçue des intervenants en santé publique. Tout au long de 2015, l'équipe d'évaluation et surveillance de la santé de la population a recueilli la rétroaction des intervenants en ce qui concerne I'utilité de renseignements hebdomadaires, mais a aussi résumé I'utilité du taux d'incidence mobile afin d'orienter les mesures de santé publique.

\section{Résultats}

\section{Activité de la coqueluche}

Cent-trente-six (136) cas de coqueluche ont été déclarés en Nouvelle-Écosse entre le 1er janvier et le 31 décembre 2015. De ceux-ci, 105 (76,5 \%) correspondaient à la définition de cas pour un cas confirmé selon une confirmation de laboratoire ou un lien épidémiologique avec un cas confirmé en laboratoire, 10 $(7,4 \%)$ étaient des cas probables et 21 (15,4\%) étaient des cas soupçonnés. L'âge des cas variait de 0 à 62 ans, et l'âge moyen était de 15,6 ans. La plus grande proportion de cas ont été déclarés chez des personnes âgées de 20 ans et plus, alors que le taux d'incidence cumulative le plus élevé a été observé chez les moins de cinq ans (tableau 1). En date du 31 décembre 2015, le taux d'incidence cumulative provincial était de 11,2 \% pour 100000 habitants (tableau 1).

\section{Tableau 1 : Nombre de cas et incidence cumulative des cas de coqueluche confirmés pour les zones de gestion de la santé et le Sud-Ouest, déclarés en Nouvelle-Écosse entre le $1^{\mathrm{er}}$ janvier et le 31 décembre 2015}

\begin{tabular}{|c|c|c|c|c|c|c|c|c|}
\hline \multirow{2}{*}{$\begin{array}{c}\text { Groupe } \\
\text { d'âge (ans) }\end{array}$} & \multirow{2}{*}{$\begin{array}{c}\text { Sud- } \\
\text { Ouest }^{1}\end{array}$} & \multicolumn{5}{|c|}{ Zone de gestion de la santé } & \multirow{2}{*}{ Population } & \multirow{2}{*}{$\mathrm{IC}^{2}$} \\
\hline & & Est & Ouest & Nord & Centre & Total & & \\
\hline 0 à 4 & 9 & 4 & 12 & 1 & 13 & 30 & 39271 & 76,4 \\
\hline 5 à 9 & 8 & 3 & 11 & 1 & 5 & 20 & 43825 & 45,6 \\
\hline 10 à 14 & 6 & 0 & 6 & 4 & 5 & 15 & 45850 & 32,7 \\
\hline 15 à 19 & 7 & 1 & 7 & 1 & 0 & 9 & 54060 & 16,6 \\
\hline Plus de 20 & 16 & 3 & 16 & 3 & 9 & 31 & 755819 & 4,1 \\
\hline Total & 46 & 11 & 52 & 10 & 32 & 105 & s.o. & s.o. \\
\hline Population & 56352 & 165727 & 196648 & 154834 & 421616 & s.o. & 938825 & s.o. \\
\hline IC $^{2}$ & 81,6 & 6,6 & 26,4 & $6,5 \%$ & 7,6 & s.o. & s.o. & 11,2 \\
\hline
\end{tabular}

Des cas ont été déclarés dans l'ensemble des quatre zones de gestion de la santé provinciales : 9,5\% (Nord), 10,5\% (Est), $30,5 \%$ (Centre) et 49,5\% (Ouest). La plus forte incidence cumulative $(26,4$ pour 100000$)$ dans la province a été déclarée dans la zone Ouest, avec $43,8 \%$ des cas provinciaux dans le Sud-Ouest. À la fin de 2015, le taux d'incidence annuel de la coqueluche dans le Sud-Ouest se chiffrait à 81,6 pour 100000 habitants.

\section{Comparaison des différents outils de surveillance}

Les méthodes traditionnelles ont fait l'objet de déclarations mensuelles. Elles ont démontré que le nombre de cas avait d'abord atteint un pic en mai 2015, puis commencé à décliner après septembre 2015. Un maximum de 20 cas confirmés ont été déclarés tous les mois (figure 1), la moyenne étant de 2,0 cas par semaine pour l'année 2015 (intervalle de 0 à 7 cas). Au niveau des zones, il y a eu une augmentation de 0,2, de 1,0, de 0,2 et 0,6 cas par semaine dans les zones de l'Est, de l'Ouest, du Nord et du Centre, respectivement. La rétroaction des intervenants en santé publique a mis en relief les difficultés liées à la stratification et à la contextualisation des faibles nombres de cas. Ils ont en outre rapporté une difficulté à déterminer les tendances temporelles par zone ou région géographique plus petite sur la base des nombres uniquement. D'après la rétroaction, des graphiques du taux d'incidence mobile hebdomadaire sur 40 jours ont été créés pour différentes échelles géographiques (province, zones, région) et groupes d'âge.

À la suite de la mise en œuvre du taux d'incidence mobile sur 40 jours dans les rapports hebdomadaires, les intervenants ont rapporté que le nouveau format était utile, car il était beaucoup plus à jour que les rapports mensuels. La statistique de l'incidence mobile sur 40 jours a fourni une représentation claire et contextuelle de l'activité de la maladie dans chaque zone, comparativement aux nombres de cas hebdomadaires. 
Figure 1 : Nombre de cas mensuels de coqueluche confirmés par zone de gestion de la santé déclarés par la Nouvelle-Écosse entre le $1^{\text {er }}$ janvier et le 31 décembre 2015

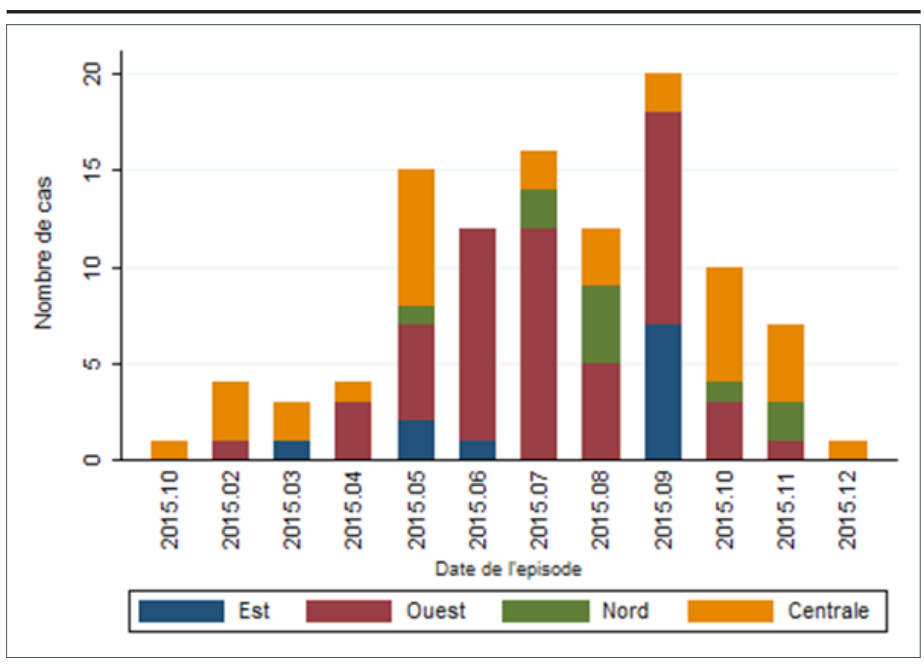

Cette statistique a été utile pour ce qui est d'explorer les tendances temporelles dans les régions zonales et sous-zonales en Nouvelle-Écosse que les données sur les nombres ne permettent pas d'explorer. Par exemple, au fil du temps, les cas de la zone Ouest (figure 2) ont produit une tendance bimodale caractérisée par des pics de l'incidence mobile en juillet et de nouveau en octobre 2015. Les cas de la zone Est ont suivi une vague unique, qui a culminé en octobre, alors que les cas de la zone Nord ont connu une augmentation plus tôt et un pic en août. L'utilité des comptes hebdomadaires était également limitée puisqu'ils ne pouvaient tenir compte de la variabilité de la taille des populations entre les zones ou les groupes d'âge. Les comptes de cas de coqueluche hebdomadaires dans la zone du Centre étaient relativement élevés comparativement aux autres zones, probablement en raison de la grande population. Toutefois, la statistique de l'incidence mobile a permis de contextualiser l'activité de la coqueluche et l'analyse

Figure 2 : Incidence mobile sur 40 jours calculée pour 100000 habitants des cas de coqueluche confirmés par zone de gestion de la santé en Nouvelle-Écosse, 2015

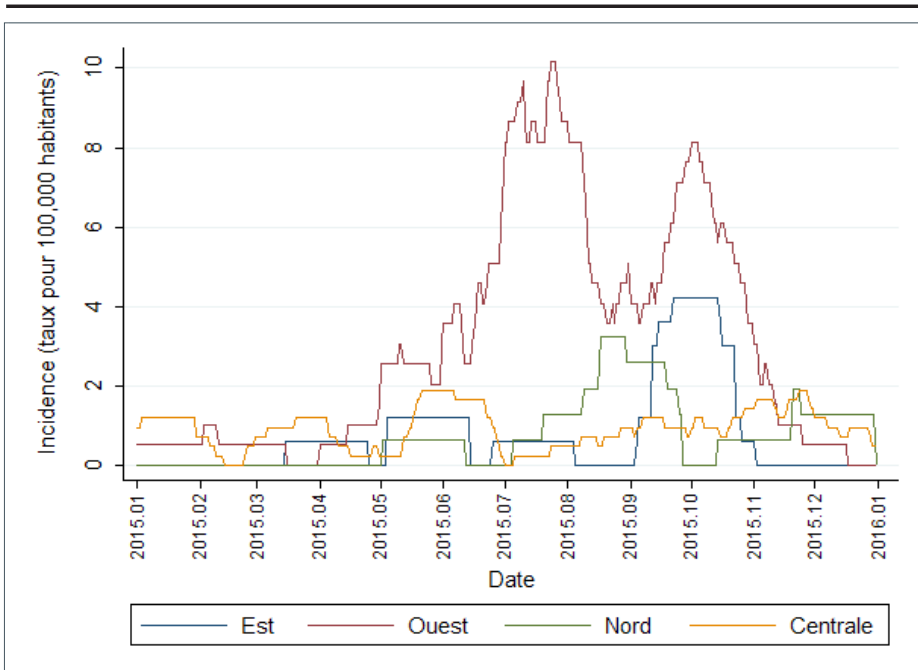

résultante a démontré que l'activité dans la zone du Centre était généralement plus faible et plus sporadique, mais sans présenter les grands pics observés dans les autres zones.

En date du $1^{\text {er }}$ juillet 2015 , le taux d'incidence cumulative pour la coqueluche était de 4,2 pour 100000 habitants; de 10,2 pour 100000 dans la zone Ouest et de 31,9 pour 100000 dans la zone Sud-Ouest. Les intervenants ont demandé une statistique plus représentative du niveau d'activité actuel, étant donné que les nombres de cas hebdomadaires ou les taux d'incidence cumulative annuels n'étaient pas utiles pour orienter la mise en œuvre des interventions à diverses échelles géographiques. Les nombres de cas étaient trop faibles pour permettre des comparaisons significatives comme ils n'étaient pas ajustés pour tenir compte de la taille de la population, tandis que I'incidence cumulative annuelle exagérait l'activité actuelle de la maladie puisque les cas non contagieux n'étaient pas exclus

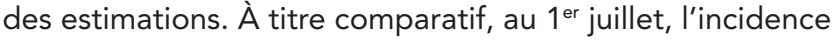
mobile sur 40 jours était de 0,5 pour 100000 habitants en Nouvelle-Écosse, de 8,1 pour 100000 dans la zone Ouest et de 24,8 pour 100000 dans la zone Sud-Ouest (figure 3). L'incidence mobile sur 40 jours a mis en lumière la nécessité d'interventions dans la zone du Sud-Ouest, comme le recommande le médecin hygiéniste local. De même, les estimations de l'incidence mobile n'appuyaient pas la mise en œuvre des mesures de santé publique à l'échelle des zones ou de la province.

Figure 3 : Incidence mobile sur 40 jours pour 100000 habitants de cas de coqueluche confirmés déclarés en Nouvelle-Écosse, dans la zone Ouest et la partie Sud-Ouest, 2015

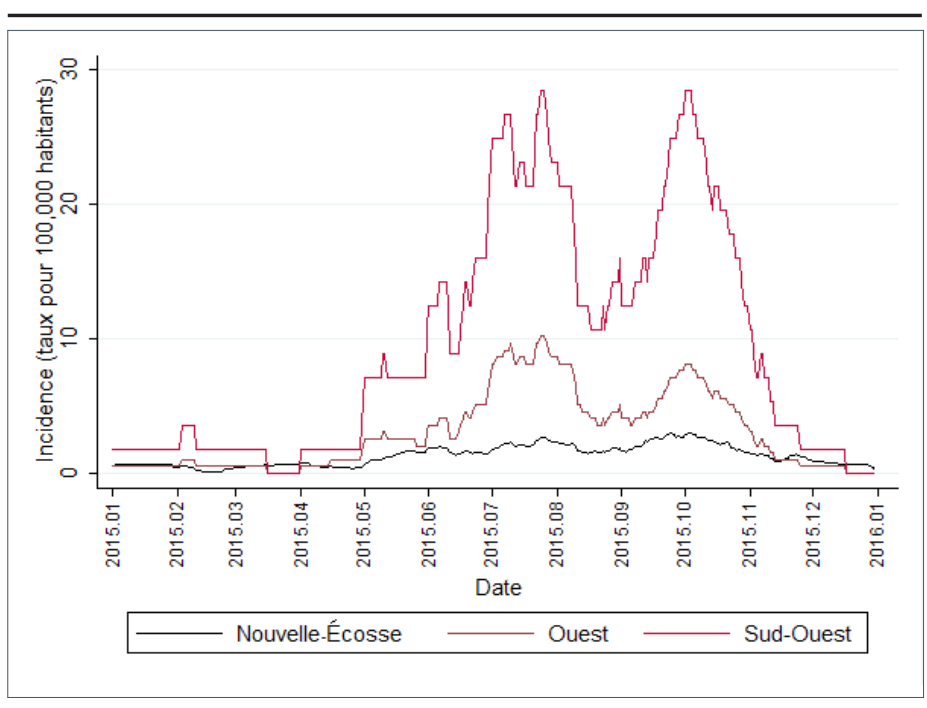

L'analyse a joué un rôle important dans l'orientation des mesures de santé publique appropriées à différentes échelles géographiques. La figure 3 présente une comparaison des taux d'incidence mobile entre trois échelles géographiques. L'activité était légèrement élevée dans l'ensemble de la province, et c'est la partie Sud-Ouest de la zone Ouest qui a enregistré les taux les plus élevés. On a demandé aux fournisseurs locaux de la partie Sud-Ouest de se concentrer sur l'immunisation des enfants afin de faire écho aux lignes directrices provinciales, en particulier pour les enfants âgés de 0 à 4 ans, et d'offrir des vaccins à toutes les femmes enceintes (plus de 26 semaines 
de grossesse), quel que soit leur état vaccinal. La dernière recommandation différait de la recommandation provinciale selon laquelle seules les femmes enceintes de 26 semaines et plus qui n'avaient pas reçu une dose de vaccin contre la coqueluche à l'âge adulte devraient être vaccinées, mais était conforme aux recommandations du Comité consultatif national de l'immunisation pour la coqueluche selon l'épidémiologie locale qui a démontré une activité accrue (26).

\section{Expérience d'intervention}

Au cours de la période d'incidence accrue de la coqueluche en Nouvelle-Écosse, la question à savoir s'il était nécessaire d'intensifier les interventions de contrôle dans la totalité ou une partie de la province a fait l'objet d'un vaste débat. L'incidence mobile sur 40 jours a donné une meilleure idée du moment auquel a eu lieu le pic d'incidence de la coqueluche et a donc éclairé la décision des autorités sanitaires publiques provinciales, c'est-à-dire que l'institution de mesures de contrôles additionnelles dans la région géographique où l'activité est la plus forte n'était pas requise à l'échelle provinciale. Les intervenants ont dit apprécier les renseignements supplémentaires fournis par le taux d'incidence mobile sur 40 jours.

\section{Discussion}

Pendant l'augmentation du nombre de cas de coqueluche déclarés en 2015, I'utilisation de l'incidence mobile sur 40 jours s'est avérée un moyen utile de visualiser les données de surveillance afin d'illustrer les tendances régionales et temporelles en Nouvelle-Écosse. II s'est ensuivi un programme de vaccination d'urgence dans une partie d'une zone de gestion de la santé en réponse à l'activité de la maladie.

Le nombre accru de cas de coqueluche en Nouvelle-Écosse tout au long de 2015 était inquiétant comme il dépassait de loin les nombres et les taux observés en moyenne et lors du pic cyclique précédent de 2012 (27). Le taux d'incidence mobile sur 40 jours a permis de bien décrire l'activité accrue.

L'utilisation d'estimations mobiles n'est pas une nouvelle technique; les moyennes mobiles sont employées dans d'autres formes de surveillance, telles que la détermination de seuils d'alerte dans la surveillance syndromique (28-30). Bien que les moyennes mobiles aient servi à établir des seuils dans le cadre d'autres recherches, cette utilisation n'était pas le but de l'étude. Un autre avantage de l'approche de l'incidence mobile est son potentiel de définir et de surveiller les seuils d'occurrence de la maladie.

On a associé deux forces principales à l'utilisation de l'incidence mobile sur 40 jours durant cet événement de santé publique. D'abord, la moyenne mobile sur 40 jours a fourni un outil visuel opportun permettant de comparer les statistiques entre différentes sous-populations, à l'aide de renseignements plus récents que ceux fournis dans les rapports mensuels sur les maladies à déclaration obligatoire. Ces comparaisons n'étaient pas possibles au moyen d'une courbe épidémiologique standard des nombres de cas. Par exemple, pour cet événement, la tendance à la hausse dans la zone Ouest ne ressortait pas aussi clairement du compte mensuel traditionnel, mais était plus évidente dans le tracé de la moyenne mobile sur 40 jours.

Ensuite, le recours à une moyenne mobile sur 40 jours a contribué à éviter certaines des complications associées à la déclaration des faibles nombres de cas et à démêler les questions derrière le taux cumulatif en hausse constante. La déclaration des nombres faibles est également à même de menacer la confidentialité des gens $(19,20)$, et les nombres faibles sont également plus affectés par le hasard. II est possible de remédier à cette instabilité en fusionnant les catégories de façon à augmenter le nombre (31) ou, comme dans cet article, en augmentant l'intervalle de temps. La Nouvelle-Écosse est une petite province comptant une population de moins d'un million de résidents. Lorsque les taux de coqueluche sont élevés, le nombre réel de cas demeure une petite quantité, en particulier lorsqu'il est stratifié par zone.

\section{Limites}

Le taux d'incidence mobile sur 40 jours comporte plusieurs limites. Bien que cette analyse ait fourni des renseignements sur l'incidence propres à la maladie aux décideurs de la santé publique, il s'agit d'une méthode qui n'est pas traditionnellement utilisée; ses résultats ne sont donc pas comparables aux taux de coqueluche dans d'autres provinces et territoires. Il existe peu de discussions publiées sur des incidents où l'on a mis en œuvre ou abandonné des interventions en santé publique sur la base de ce type d'analyse. Au Nouveau-Brunswick, la fin d'une éclosion de coqueluche a été déclarée lorsque l'activité est revenue dans les limites de deux écarts-types d'une moyenne mobile lissée sur cinq ans pour au moins deux semaines (28). Cependant, il s'agit là d'un délai beaucoup plus long que celui utilisé dans l'analyse. L'utilisation de périodes de transmissibilité pour définir des intervalles de temps pourrait donner une indication de la transmission communautaire actuelle, sans toutefois considérer le facteur additionnel de la nature cyclique connue de la maladie, dont les pics peuvent durer plusieurs mois et réapparaître sur une période de trois à cinq ans. Un délai plus long peut être nécessaire afin de définir les seuils optimaux pour la mise en œuvre ou l'abandon d'interventions de contrôle communautaires. En outre, comme le nombre de cas est très faible, la visualisation des données pourrait en être faussée. Par exemple, la caractéristique bimodale observée dans cette analyse pourrait être un bruit aléatoire, un artéfact du processus de la moyenne mobile. Par conséquent, il faut interpréter les graphiques avec prudence lorsqu'ils reflètent de faibles nombres.

L'utilisation de la date d'épisode constitue une autre faiblesse de cette analyse. En Nouvelle-Écosse, la date d'épisode correspond à la date d'apparition des symptômes. La définition de la date d'épisode peut varier selon les administrations et représenter la date de déclaration, la date de confirmation en laboratoire ou la date d'apparition des symptômes. À ce titre, l'utilité de la comparaison de l'incidence mobile d'une administration à l'autre peut être limitée.

L'intervalle de temps de 40 jours pourrait ne pas être l'intervalle le plus approprié ou le plus précis pour représenter le potentiel infectieux de la coqueluche en Nouvelle-Écosse. Les données indiquent que la plus longue période d'incubation pourrait être de 42 jours (2); un tel délai pourrait donc constituer une représentation plus complète de la transmissibilité potentielle. 
Sinon, l'utilisation de la période d'incubation moyenne de 10 jours pourrait également renseigner sur la propagation probable de la maladie. Des recherches et des analyses supplémentaires sont nécessaires afin de valider l'intervalle de temps optimal pour la déclaration de l'incidence mobile. La transmissibilité de la coqueluche dépend du stade de la maladie et de si un patient est traité. Les cas sont les plus contagieux au stade catarrhal et au début du stade paroxystique (les deux premières semaines), après quoi la transmissibilité décroît graduellement au cours des trois semaines suivantes $(1,2)$. De plus, les cas ne sont plus contagieux au bout de cinq jours de traitement avec des antibiotiques. Durant cet événement de santé publique, plusieurs cas dans des familles ont été diagnostiqués rétrospectivement. Ces cas rétrospectifs n'ont probablement pas bénéficié du traitement et ont donc probablement été transmissibles pendant des intervalles de plus longue durée. La période de transmissibilité a été présumée la même pour tous les cas compris dans les calculs de l'incidence mobile sur 40 jours. Les cas qui ont reçu un traitement comme ceux qui ont continué de répandre la maladie ont été inclus. Qui plus est, les retards dans la déclaration des cas restreignent l'efficacité en temps réel de cette méthode.

Malgré les limites de la méthodologie, l'incidence mobile peut être utilisée en combinaison avec des méthodes de surveillance des maladies transmissibles traditionnelles, surtout dans les cas qui concernent de rares événements de santé publique, dont les périodes d'incubation sont longues. Ces méthodes ont le potentiel d'être employées dans des situations similaires puisqu'elles complètent les méthodes épidémiologiques traditionnelles pour la surveillance des maladies à déclaration obligatoire. Dorénavant, l'incidence mobile pourrait servir à définir et à surveiller les seuils d'occurrence de la maladie. II s'agit d'un domaine que les autorités en matière de santé publique devront considérer à mesure qu'elles poursuivent leurs efforts pour améliorer la surveillance des maladies transmissibles. Par conséquent, il faut interpréter les graphiques avec prudence lorsqu'ils reflètent de faibles nombres.

\section{Conclusion}

Les taux d'incidence mobile sur 40 jours ont fourni un moyen utile de décrire et de comparer les tendances de la coqueluche en Nouvelle-Écosse pour des échelles géographiques et des groupes d'âge différents. Le taux d'incidence mobile sur 40 jours s'est révélé un outil supplémentaire utile pour étudier l'activité de la coqueluche et éclairer les décisions des épidémiologistes, des médecins hygiénistes et des gestionnaires des maladies transmissibles locaux en matière de santé publique. En outre, ces résultats ont contribué à orienter les mesures de santé publique, en particulier dans les régions de forte activité de la coqueluche. Les méthodes présentées dans le présent document pourraient être adaptées afin d'étudier et de gérer d'autres maladies transmissibles.

\section{Remerciements}

Les auteurs remercient le personnel de santé publique local qui a recueilli et déclaré les données employées dans l'analyse et les intervenants qui ont fourni de la rétroaction aux fins de cette évaluation.

\section{Conflit d'intérêts}

Aucun.

\section{Financement}

Cette recherche a été appuyée par le ministère de la Santé et du Mieux-être de la Nouvelle-Écosse et le Programme canadien d'épidémiologie de terrain.

\section{Références}

1. Heymann DL. Control of communicable diseases manual, 19th ed. Washington, DC: American Public Health Association; 2008.

2. Agence de la santé publique du Canada [Internet]. La coqueluche (toux coquelucheuse) Professionnels de la santé. Ottawa, ON: ASPC; 2014 [mis à jour le 21 fév 2014; consulté le 9 août 2015]. Disponible à l'adresse : http:// www.phac-aspc.gc.ca/im/vpd-mev/pertussis/professionalsprofessionnels-fra.php.

3. Department of Health and Wellness. Nova Scotia communicable disease manual [Internet]. Halifax, NS: Nova Scotia Department of Health and Wellness; 2001 [consulté le 9 août 2015]. Disponible à l'adresse : http://novascotia.ca/ dhw/cdpc/cdc/documents/Pertussis.pdf.

4. Gilley M, Goldman RD. Protecting infants from pertussis. Can Fam Phys. 2014 Feb;60(2):138-40.

5. Pimentel AM, Baptista PN, de Alencar Ximenes RA, Rodrigues LC, Magalhães V, Silva AR, Souza NF, Matos DG, Pessoa AK; Pert - Pertussis Study Group. Pertussis may be the cause of prolonged cough in adolescents and adults in the interepidemic period. Braz J Infect Dis. 2015;19(1):43-46.

6. Wood N, Mclntyre P. Pertussis: Review of epidemiology, diagnosis, management and prevention. Paed Resp Rev. 2008;(9):201-211.

7. Agence de la santé publique du Canada. Couvertures vaccinale des enfants au Canada; Résultats de l'enquête sur la couvertures vaccine ale nationale des enfants (ECVNE) de 2013 [Internet]. Ottawa, ON: ASPC; 2016 [consulté le 24 août 2016]. Disponible à l'adresse : http://healthycanadians. gc.ca/publications/healthy-living-vie-saine/immunizationcoverage-children-2013-couverture-vaccinale-enfants/alt/ icc-2013-cve-fra.pdf.

8. Population Health Assessment and Surveillance. Schoolbased immunization coverage Nova Scotia 2013-2014 [Internet]. Halifax, NS: Nova Scotia Department of Health and Wellness; 2015 [consulté le 20 juill 2015]. Disponible à l'adresse : http://novascotia.ca/dhw/populationhealth/ documents/School-Based-Immunization-Coverage-NovaScotia-2013-2014.pdf. 
9. Bolotin S, Harvill ET, Crowcroft NS. What to do about pertussis vaccines? Linking what we know about pertussis vaccine effectiveness, immunology and disease transmission to create a better vaccine. Pathog Dis. 2015 Nov 1; 73(8):ftv057.

10. Riolo MA, Rohani P. Combating pertussis resurgence: One booster vaccination schedule does not fit all. Proc Natl Acad Sci USA. 2015 Feb 3;112(5):E472-7.

11. Van Rie A, Hethcote HW. Adolescent and adult pertussis vaccination: Computer simulations of five new strategies. 2004 Aug 13;22(23-24):3154-65.

12. Cornia PB, Hersh AL, Lipsky BA, Newman TB, Gonzales, R. Does this coughing adolescent or adult patient have pertussis? JAMA. 2010 Aug 25;304(8):890-6.

13. Skowronski DM, De Serres G, MacDonald D, Wu W, Shaw C, Macnabb J, Champagne S, Patrick DM, Halperin SA. The changing age and seasonal profile of pertussis in Canada. J Infect Dis. 2002 May 15;185(10):1448-53.

14. Tsang RS, Shuel M, Jamieson FB, Drews $S$, Hoang L, Horsman G, Lefebvre B, Desai S, St-Laurent M. Pertactin-negative Bordetella pertussis strains in Canada: Characterization of a dozen isolates based on a survey of 224 samples collected in different parts of the country over the last 20 years. Int J Infect Dis. 2014 Nov;28:65-9.

15. Smith T, Rotondo J, Desai S, Deehan H. Surveillance de la coqueluche au Canada: tendances jusqu'à 2012. Relevé des maladies transmissibles au Canada. 2014; 40(3):21. Disponible à l'adresse : http://www.phac-aspc.gc.ca/ publicat/ccdr-rmtc/14vol40/dr-rm40-03/dr-rm40-03-per-fra. php.

16. Department of Immunizations, Vaccines and Biologicals. WHO-recommended standard for surveillance of select vaccine preventable diseases [Internet]. Geneva: World Health Organization; 2003 [mis à jour juill 2005; consulté le 28 août 2016]. Disponible à l'adresse : http://apps.who. int/iris/bitstream/10665/68334/1/WHO_V-B_03.01_eng. pdf?ua $=1$.

17. Gouvernement du Canada [Internet]. Maladies à declaration obligatoire en direct. Ottawa: Gouvernement du Canada; 2016 [mis à jour le2016 Jun 28; consulté le 28 août 2016]. Disponible à l'adresse : http://maladies.canada.ca/ declaration-obligatoire/.

18. Doherr MG, Audigé L. Monitoring and surveillance for rare health-related events: A review from the veterinary perspective. Philos Trans R Soc Lond B Biol Sci. 2001 Jul 29;356(1411):1097-106.

19. Statistics Canada. Disclosure control. In Statistics Canada quality guidelines, 4th Ed [Internet]. Ottawa, ON: Statistics Canada; 2003 [mis à jour le 10 avril 2014; consulté le 24 août 2016]. Disponible à l'adresse : http://www.statcan.gc.ca/ pub/12-539-x/12-539-x2003001-eng.pdf.

20. National Association of Health Data Organizations. NAHDOCDC Cooperative Agreement Project. Statistical approaches for small numbers: Addressing reliability and disclosure risk. [internet] Salt Lake City, UT: The Association; 2004 [mis à jour le2005 April 5; consulté le 9 août 2015]. Disponible à l'adresse : https://www.nahdo.org/sites/nahdo.org/files/ Data_Release_Guidelines.pdf.
21. Office of the Chief Medical Officer of Health. Pertussis outbreak investigation report [Internet]. Fredericton, NB: New Brunswick Department of Health; 2014 Apr [consulté le 9 août 2015]. Disponible à l'adresse : http://www2. gnb.ca/content/dam/gnb/Departments/h-s/pdf/en/CDC/ HealthProfessionals/PertussisReport.pdf.

22. Population Health Assessment and Surveillance. Nova Scotia surveillance guidelines for notifiable diseases and conditions: Pertussis [Internet]. Halifax, NS: Nova Scotia Department of Health and Wellness; 2015 [consulté le 20 juill 2015]. Disponible à l'adresse : http://novascotia.ca/dhw/ populationhealth/surveillanceguidelinesépertussis.pdf.

23. Nova Scotia Public Health. Surveillance guidelines. In Application for notifiable disease surveillance (ANDS) Business procedures document [Internet]. Halifax, NS: Nova Scotia Department of Health and Wellness; 2015 [consulté le 10 juin 2016]. Disponible à l'adresse : http://novascotia. $\mathrm{ca} / \mathrm{dhw} /$ populationhealth/surveillanceguidelines/ANDS_ Business_Procedures.pdf.

24. Stata Corp. Stata statistical software: Release 13 [computer program]. College Station, TX: StataCorp LP; 2013.

25. Statistiques Canada [Internet]. CANSIM Table 051-0001 2012-2016 Estimates of population, by age group and sex for July 1, Canada, provinces and territories. Ottawa, ON: Statistics Canada; 2015 [consulté le 2015 Sep 28]. Available from http://www5.statcan.gc.ca/cansim/a26?lang=eng\&retrL ang =eng\&id $=0510001 \& \&$ pattern $=\& s t B y V a l=1 \& p 1=1 \& p 2=3$ 7\&tabMode $=$ dataTable\&csid.

26. Agence de la santé publique du Canada[Internet]. Une déclaration d'un comité consultatif (DCC), Comité consultatif nationale de l'immunisation (CCNI). Mise à jour sur la vaccination contre la coqueluche pendant la grossesse. Ottawa, ON: I'Agence; 2014 [consulté le 24 août 2016]. Disponible à l'adresse : http://publications.gc.ca/collections/ collection_2014/aspc-phac/HP40-93-2014-fra.pdf.

27. Nova Scotia Health and Wellness. Notifiable diseases in Nova Scotia 2012 surveillance report: Population health assessment and surveillance [Internet]. Halifax, NS: Nova Scotia Department of Health and Wellness; 2013 [consulté le 20 juill 2015]. Disponible à l'adresse : http://novascotia. $\mathrm{ca} / \mathrm{dhw} /$ populationhealth/documents/Annual-NotifiableDisease-Surveillance-Report-2012.pdf.

28. Brown SM, Benneyan JC, Theobald DA, Sands K, Hahn MT, Potter-Bynoe GA, Stelling JM, O'Brien TF, Goldmann DA. Use of binary cumulative sums and moving averages in nosocomial infections cluster detection. Emerg Infect Dis. 2002 Dec;8(12):1426-32.

29. Allard R. Use of time-series analysis in infectious disease surveillance. Bull World Health Organ. 1998;76(4):327-33.

30. Edge VL, Pollari F, Lim G, Aramini J, Sockett P, Martin SW, Wilson J, Ellis A. Syndromic surveillance of gastrointestinal illness using pharmacy over-the-counter sales: A retrospective study of waterborne outbreaks in Saskatchewan and Ontario. Can J Public Health. 2004 Nov-Dec;95(6):446-50.

31. Takiar R, Nadayil D, Nandakumar A. Problem of small numbers in reporting of cancer incidence and mortality rates in Indian cancer registries. Asian Pac J Cancer Prev. 2009 Oct-Dec;10(4):657-60. 